\title{
MODELING THE GLOBAL OSCILLATIONS OF EPSILON ERIDANI
}

\author{
David R. Soderblom \\ Space Telescope Science Institute \\ 3700 San Martin Drive \\ Baltimore, Maryland 21218 USA
}

\author{
Werner Däppen \\ High Altitude Observatory \\ National Center for Atmospheric Research \\ P.O. Box 3000 \\ Boulder, Colorado 80307 USA
}

\begin{abstract}
We have reviewed the observational data for $\epsilon$ Eri to derive our best estimates of luminosity, radius, temperature, composition, and age, as well as the uncertainties associated with each of these. These quantities are then used in stellar structure models to try to reproduce the global oscillation frequencies observed by Noyes et al. We find that we can reproduce the observed frequencies and splittings at least as well as the computations of Guenther and Demarque, yet for significantly different stellar parameters, most notably the age.
\end{abstract}

\section{REVIEW OF THE OBSERVATIONAL DATA}

Noyes et al. (1984) have recently reported the tentative detection of global $p$-mode oscillations, akin to the Sun's 5-minute oscillations, on the K2V star $\epsilon$ Eridani. Guenther and Demarque (1986: G\&D) applied stellar structure models to those observations, to reproduce both the observed frequencies and the frequency spacing of the observations. Their results warrant independent verification. Moreover, the fundamental stellar parameters (radius, temperature, luminosity, metallicity, and age) that are necessary inputs to such models have, we believe, values or uncertainties that are rather different from those used by G\&D.

A full description of our data and results will be published elsewhere; we present here only a brief summary. We began by reviewing the available photometry for $\epsilon$ Eri, and found that its magnitudes and colors were much less certain that expected for a well-observed star. Johnson et al. (1966) tabulate individual photometric observations. Using only their best data we derive $V=3.728 \pm 0.039,(U-B)=0.579 \pm 0.048,(B-V)=0.885 \pm 0.024$, and $(V-R)=0.718 \pm 0.021$. These large uncertainties are not due to starspots or rotational modulation of the colors, because the changes in magnitude and colors are uncorrelated. 
The parallax of $\epsilon$ Eri is $\pi=0.3046 \pm 0.0046 \operatorname{arcsec}(v a n$ Altena 1985). This then means $M_{\mathrm{V}}=6.147 \pm 0.085$. The bolometric correction for the above colors is either -0.207 (Johnson 1966) or -0.315 (Hayes 1978).

The derived effective temperature of $\epsilon$ Eri is more consistent. From either the calibration of Johnson (1966) or Popper(1980), the above colors give $T_{\text {eff }}=5000 \pm 50 \mathrm{~K}$. The temperature and luminosity then yield the star's radius.

Three spectroscopic determinations of the metallicity of $\epsilon$ Eri have been made (Cayrel de Strobel et al. 1985). Their mean is $[\mathrm{Fe} / \mathrm{H}]=-0.23$, or $Z=0.010$. There may be a systematic underestimate of the metallicity, due to $\epsilon$ Eri's chromospheric activity, so that it is not possible to rule out the possibility that its abundances are roughly solar.

G\&D consider the age of $\epsilon$ Eri to be a free parameter in their analysis, but we feel that it is, in fact, one of the better determined properties of this star. They note, correctly, that a star's metallicity is only roughly related to age: one may safely conclude that a star with $[\mathrm{Fe} / \mathrm{H}] \lesssim-1$ is old, but young clusters (e.g., Coma) exist with metallicities less than the Sun's. Similarly, $\alpha$ Centauri is an example of a star of the same age as the Sun, but about 50metallicity (Furenlid and Kurucz 1986). G\&D are incorrect, however, in stating that the strength of the chromospehric emission is also only a staistical age indicator. Epsilon Eri is unarguably an active star: it has strong $\mathrm{Ca}$ II $\mathrm{H}$ and $\mathrm{K}$ emission (Noyes et al. 1984), strong UV emissions (Kelch 1978; Linsky et al. 1982), and strong X-ray emission (Johnson 1981). These are all indicative of the strong stellar magnetic fields associated with youth. Duncan, Soderblom, and Johnson (1986) have recently substantiated Skumanich's (1972) power-law relation between $\mathrm{HK}$ emission and age for a broad range of ages, demonstrating that all active stars (save close binaries) are necessarily young.

Using this $t^{-1 / 2}$ law, one derives an age of $0.75 \mathrm{Gyr}$ for $\epsilon$ Eri. It is sufficient to make the more conservative statement that $t(\epsilon$ Eri $)<1 \mathrm{Gyr}$, i.e., it is essentially on the Zero Age Main Sequence. The age that G\&D derive, $12 \mathrm{Gyr}$, cannot be reconciled with this.

The values we derive for the physical parameters of $\epsilon$ Eri are summarized in Table I, where G\&D's values are given for comparison.

\section{MODELS OF EPSILON ERIDANI}

Most of the differences in Table I between this work and G\&D are not significant, although we have generally found larger uncertainties for the various quantities. The fact that G\&D left the stellar radius as a free parameter is also not important, since it is naturally constrained by their choices of temperature and luminosity. The one significant difference is that G\&D take $\epsilon$ Eri's age to be a free parameter, whereas we wish to fit the socillation spectrum with a model appropriate to a young star. We leave the mass $(\mathcal{M})$ and mixing length parameter $\left(\alpha=\ell / H_{p}\right)$ to be determined.

The calculations are based on a version of the Geneva Observatory evolution code, modified by one of us (W.D.). This code uses a free-energy-minimization equation of state, the Böhm-Vitense mixing length formalism, and Cox-Stewart opacities. The pulsation code is from Christensen-Dalsgaard. Several of the models of G\&D were recomputed, and we found good agreement both in the oscillation spectrum and the evolutionary properties.

We then calculated the oscillation spectrum for values of $\alpha$ and $\mathcal{M}$ near 0.5 and 0.8 , 
respectively, which reproduce the global properties (temperature, luminosity, and radius) of $\epsilon$ Eri, for an age of $1 \mathrm{Gyr}$. These models were then adjusted slighly in mass to force $\Delta \nu_{0}=172 \mu \mathrm{Hz}$. In Table II, we compare several of our models to the observations and to G\&D's Model 7. Our models produce a better overall fit, and $\alpha=0.50$ is probably the best match to the observations, although all three are adequate fits.

TABLE I: Summary of Model Inputs for Epsilon Eridani

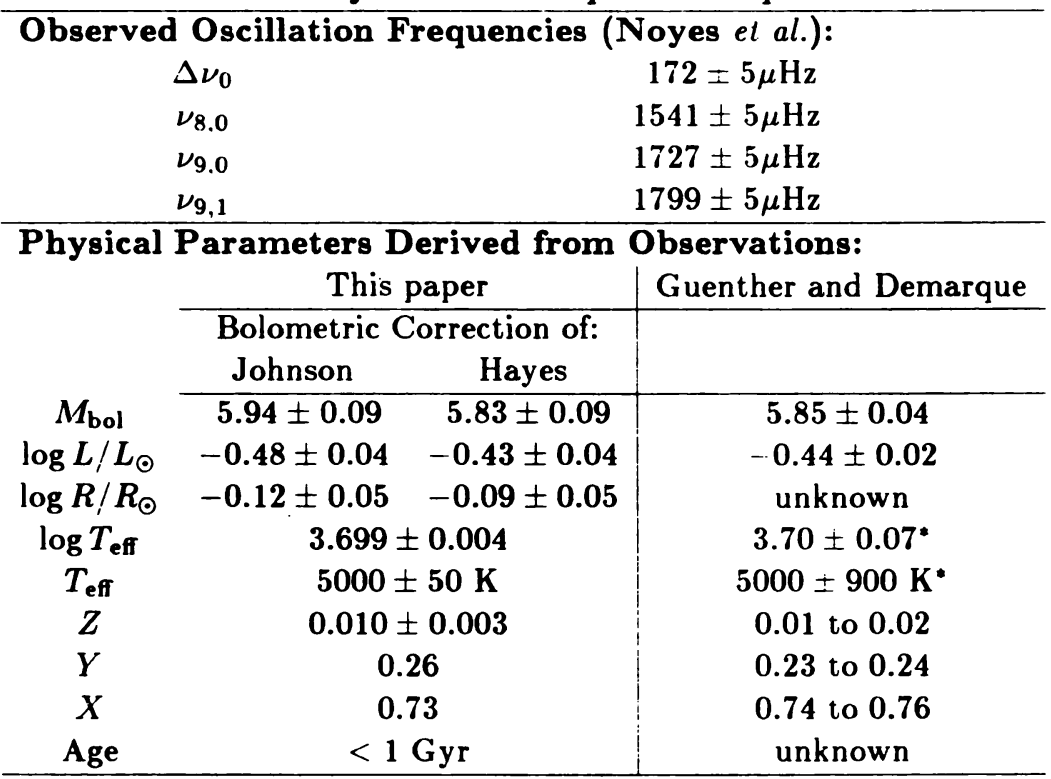

* This large uncertainty for the temperature is presumably a typographical error in G\&D, and should probably be 0.007 dex or $80 \mathrm{~K}$.

TABLE II: Summary of Model Results for Epsilon Eridani

\begin{tabular}{lrrrrrr}
\hline & $\nu_{8,0}$ & $\nu_{9,0}$ & $\nu_{9,1}$ & $\delta \nu_{8,0}$ & $\delta \nu_{9,0}$ & $\delta \nu_{9,1}$ \\
\hline Observed (Noyes et al.) & 1541 & 1727 & 1799 & - & - & - \\
G\&D Model 7 & 1567 & 1741 & 1819 & -26 & -14 & +20 \\
This paper, $\alpha=0.50, \mathcal{M}=0.77$ & 1548 & 1721 & 1800 & -7 & +6 & -1 \\
$\alpha=0.55, \mathcal{M}=0.78$ & 1545 & 1719 & 1798 & -4 & +8 & +1 \\
$\alpha=0.60, \mathcal{M}=0.80$ & 1550 & 1724 & 1803 & -9 & +3 & -4 \\
\hline
\end{tabular}




\section{COMMENTS AND CONCLUSIONS}

We have computed stellar models that produce an excellent fit to the observed frequency spectrum of $\epsilon$ Eridani, and which also fit its other physical properties as well. Our aim was to construct such models for stars near the ZAMS, out of a conviction that $\epsilon$ Eri could not possibly be as old ( $12 \mathrm{Gyr}$ ) as G\&D determined. We find that ZAMS models work at least as well as those used by G\&D in matching the observations, although some may find our low values of $\alpha$ (near 1/2) as distasteful as we find an old age.

Given that models with such different parameters can produce adequate matches to the observations, our primary conclusion is that the basic physical parameters of a star (mass, metallicity, temperature, luminosity, radius, and age) must be constrained in order to be able to interpret observations of oscillations. Detecting the low-degree splitting $(\ell=0 v s . \ell=2)$ can overcome this problem to some extent, but is observationally even more difficult than detecting the oscillations, because this splitting is only about $10 \mu \mathrm{Hz}$, and is near the $11.6 \mu \mathrm{Hz}$ diurnal sidelobe. We feel that stars in nearby visual binaries are probably the best potential targets for asteroseismology because their masses are known. Even for these stars serious supplemental efforts are needed to determine metallicities, parallaxes, etc., because rough values won't do.

\section{REFERENCES}

Cayrel de Strobel, G., Bentolila, C., Hauck, B., and Duquennoy, A. 1985, Astron. Astrophys. Suppl., 59, 145.

Duncan, D.K., Soderblom, D.R., and Johnson, D.R.H. 1986, in preparation.

Furenlid, I., and Kurucz, R. 1986, private communication.

Guenther, D.B., and Demarque, P. 1986, Astrophys. J., 301, 207.

Hayes, D. 1978, in The HR Diagram, IAU Symp. 80, edited by A.G.D. Philip and

D.S. Hayes, (Dordrecht: Reidel), p. 65.

Johnson, H.L. 1966, Ann. Rev. Astron. Astrophys., 4, 193.

Johnson, H.L., Mitchell, R.I., Iriarte, B., and Wiśniew'ski, Z. 1966, Comm. Lunar Planet. Lab., 4, 99.

Johnson, H.M. 1981, Astrophys. J., 243, 234.

Kelch, W.L. 1978, Astrophys. J., $222,931$.

Linsky, J.L., Bornmann, P.L., Carpenter, K.G., Wing, R.F., Giampapa, M.S., Worden,

S.P., and Hege, E.K. 1982, Astrophys. J., 260, 670.

Noyes, R.W., Baliunas, S.L., Belserene, E., Duncan, D.K., Horne, J., and Widrow, L. 1984, Astrophys. J. Lett., 285, L23.

Popper. D.M. 1980, Ann. Rev. Astron. Astrophys., 18, 115.

Skumanich, A. 1972, Astrophys. J., 171, 565.

van Altena, W. 1985, private communication. 